\title{
Sonoporation using microbubble BR14 promotes pDNA/siRNA transduction to murine heart
}

\author{
Sei Tsunoda ${ }^{a, b, c}$, Osam Mazda ${ }^{c, *}$, Yohei Oda ${ }^{a, b}$, Yasunori Iida ${ }^{a, b, c}$, Satoshi Akabame ${ }^{a, b}$, \\ Tsunao Kishida ${ }^{c}$, Masaharu Shin-Ya ${ }^{c}$, Hidetsugu Asada ${ }^{c}$, Satoshi Gojo ${ }^{d}$, Jiro Imanishi ${ }^{c}$, \\ Hiroaki Matsubara $^{\mathrm{a}}$, Toshikazu Yoshikawa ${ }^{\mathrm{b}}$ \\ a Department of Molecular Cardiology and Vascular Regenerative Medicine, Graduate School of Medical Science, \\ Kyoto Prefectural University of Medicine, Kyoto 602-8566, Japan \\ ${ }^{\mathrm{b}}$ Department of Inflammation and Immunology, Graduate School of Medical Science, Kyoto Prefectural University of Medicine, \\ Kyoto 602-8566, Japan \\ c Department of Microbiology, Graduate School of Medical Science, Kyoto Prefectural University of Medicine, Kyoto 602-8566, Japan \\ d Department of Cardiovascular Surgery, Saitama Medical Center, Kawagoe, Saitama 350-8550, Japan
}

Received 28 July 2005

Available online 18 August 2005

\begin{abstract}
Naked plasmid DNA (pDNA) and short interfering RNA (siRNA) duplexes were transduced into adult murine heart by means of sonoporation using the third-generation microbubble, BR14. Plasmid DNAs carrying luciferase, $\beta$-galactosidase ( $\beta$-gal), or enhanced green fluorescent protein (EGFP) reporter genes were mixed with BR14 and injected percutaneously into the left ventricular (LV) cavity of C57BL/6 mice while exposed to transthoracic ultrasound at $1 \mathrm{MHz}$ for 60 s. Sonoporation at an output intensity of $2.0 \mathrm{~W} / \mathrm{cm}^{2}$ and a $50 \%$ pulse duty ratio resulted in the highest luciferase expression in the heart. Histological examinations revealed significant expression of the $\beta$-gal and EGFP reporters in the subendocardial myocardium of LV. Intraventricular co-injection of siRNA-GFP and BR14 with concomitant ultrasonic exposure resulted in substantial reduction in EGFP expression in the coronary artery in EGFP transgenic mice. The present method may be applicable to gain-of-function and loss-of-function genetic engineering in vivo of adult murine heart.
\end{abstract}

(C) 2005 Elsevier Inc. All rights reserved.

Keywords: Naked plasmid DNA; Heart; Sonoporation; Microbubble; Gene transfer; RNA interference; Ultrasound; Gene therapy; Molecular therapy

Genetic modification of the heart may provide a powerful molecular tool for improving our understanding of cardiac diseases. Moreover, a technology that enables safe and efficient gene delivery to the heart may provide a novel therapeutic modality to control heart disorders. Some studies recently demonstrated that efficient transfer of genes into murine heart can be achieved by intracoronary infusion of adenoviral or adeno-associated

\footnotetext{
* Corresponding author. Fax: +81 752515331.

E-mail address: mazda@koto.kpu-m.ac.jp (O. Mazda).
}

viral vectors followed by transient aortic occlusion $[1,2]$. Viral vector-mediated procedures, however, may induce complications associated with recombinant viruses, hindering clinical application of the systems to gene therapy for cardiovascular diseases. Although non-viral methods are free from virus-associated adverse effects, their transduction efficiency is low. For example, following direct intramyocardial injection of plasmid DNA into the heart of mice [3], rats [4,5], and hamsters [6], the transgenes were expressed only within a small area surrounding the injection site. 
A more efficient non-viral method of gene transfer is sonoporation of cells. In sonoporation, ultrasound is used to increase the porosity of the cell membrane [7]. The ultrasound induces the formation of cavitation bubbles that by mechanical action cause enough damage to the cell membrane to allow large molecules in the surrounding medium to enter the cell, but not so much damage that the cell cannot reseal the membrane and survive [8]. The extent of cavitation bubble formation and subsequent increase in membrane permeability can be enhanced by use of microbubble echo contrast agents. This method has been applied to skeletal muscle of mice [9], as well as myocardium of both rats [10] and dogs [11], in vivo, using various microbubble agents including Levovist, Hexabrix, and Optison [9-18]. It has been indicated that the transfection efficiency is dependent on both the ultrasound parameters and the formulation of microbubbles.

BR14 (Bracco Research SA, Geneva, Switzerland) is a recently developed ultrasound contrast agent, which has some advantages over other echo contrast agents $[19,20]$, because it consists of stabilized relatively small microbubbles and is transiently retained within capillaries. These qualities mean that BR14 may be a better agent in sonoporation-mediated transfection. Indeed, recent studies have indicated that BR14 effectively enhanced sonoporation-based gene transfection into hepatic cancer implant in mice [21] as well as into saphenous vein graft in porcine [22].

RNA interference (RNAi) is a powerful means of analyzing the function of genes in basic researches, while the technology may also be quite useful in developing therapeutic molecular targeting strategies for treatment of diseases. RNAi was first discovered in the nematode Caenorhabditis elegans as a response to double-stranded RNA, which induced sequence-specific silencing of gene expression [23]. In mammalian cells, short interfering RNA (siRNA) 21-23 nucleotide pairs in length silences the gene with the homologous sequence $[23,24]$. The in vivo effectiveness of siRNA-mediated silencing was also studied in various organs, including the liver $[25,26]$ and the skeletal muscle [27]. However, in vivo RNAi in cardiac tissues has not been reported so far.

In the present study, we investigated whether BR14 facilitates sonoporation-mediated transfection of naked plasmid DNA into the adult murine heart. Moreover, we applied this method to transfer synthetic siRNA duplex, to knock down targeted genes in the heart in vivo.

\section{Materials and methods}

Animals. Female C57BL/6 mice (16.8 \pm 0.7 weeks old, weighing $24.0 \pm 0.5 \mathrm{~g})$ were purchased from Shimizu Laboratory Suppliers (Kyoto, Japan). Enhanced green fluorescent protein (EGFP) transgenic mice (TGM) $(27.3 \pm 4.8$ weeks old, weighing $25.3 \pm 1.1 \mathrm{~g})$ were purchased from Charles River Japan (Yokohama, Japan). All the animal experiments were performed according to the approved guidelines of Kyoto Prefectural University of Medicine.

Plasmid vectors and siRNA. The plasmids pGEG.GL3 [28], pGEG.EGFP [29], and pGEG. $\beta$ [30] carried GL3 firefly luciferase (Luc), EGFP, and Escherichia coli $\beta$-galactosidase ( $\beta$-gal) genes, respectively, under the control of the CAG promoter. Each plasmid also contained Epstein-Barr virus (EBV) nuclear antigen 1 (EBNA1) gene and EBV oriP sequence [31]. Plasmids were purified using Qiagen MegaPrep Endo-free kits (Qiagen, Hilden, Germany). siRNA duplex targeting GFP (siRNA-GFP) was purchased from Dharmacon (Lafayette, CO, USA).

In vivo experiments. Plasmid DNA $(500 \mu \mathrm{g})$ or siRNA $(40 \mu \mathrm{g})$ was diluted in $400 \mu \mathrm{l}$ PBS and mixed with $100 \mu \mathrm{l}$ BR14 microbubble. Mice were anesthetized by an intraperitoneal injection of sodium pentobarbital $(40 \mu \mathrm{g} / \mathrm{g}$ body weight), and tracheotomy was performed in the supine position to provide ventilation via a ventilator (MiniVent 845; Hugo Sachs Elektronik, March-Hugstetten, Germany) at the rate of 150 cycles per min. An incision was made in the greater and smaller pectoral muscles, and the microbubble/nucleic acid solution was injected over a period of about $10 \mathrm{~s}$ into the left ventricular (LV) cavity via the intercostal muscle using a 27 -gauge needle. The same solution was injected into the tail vein (TV) of another group of mice. At the same time as the initiation of the injection, transthoracic ultrasound insonation (sonication) was performed through a $6-\mathrm{mm}$ diameter probe with an input frequency of $1 \mathrm{MHz}$, an output intensity of 1.0 $2.0 \mathrm{~W} / \mathrm{cm}^{2}$, a pulse duty ratio (PDR) of $10-50 \%$, and a duration of 60 s. A Sonitron 2000 (Rich-Mar, Inola, OK, USA) was used to generate the ultrasound. Hydrodynamics-based transduction was performed as described previously [28]. Briefly, $40 \mu \mathrm{g}$ siRNA-GFP was diluted in $1600 \mu \mathrm{PBS}$ and injected intravenously within $4 \mathrm{~s}$ via the tail vein using a 27 -gauge needle.

Echocardiography and ECG monitoring. Transthoracic echocardiography was performed using an ultrasound platform (Nemio 30, Toshiba Medical, Tokyo, Japan) equipped with a 13-MHz imaging transducer. Under anesthesia, a parasternal view was obtained and M-mode images of the LV were recorded. Electrocardiographic monitoring was performed by limb lead (II) during the sonoporation.

Luciferase assay. Biventricular muscle was minced with a pair of scissors and homogenized in $200 \mu$ of reporter lysis buffer (Promega, Madison, WI, USA) using a sonicator. The extract was centrifuged at $14,000 \mathrm{~g}$ for $15 \mathrm{~min}$, and the Luc activity in the supernatant was measured as described [32]. Organs other than the heart were also treated as described above. Protein concentration in the extract was determined as described previously [28].

$X$-gal staining. The heart was fixed with $4 \%$ paraformaldehyde and dehydrated in sucrose solution. The specimens were then stained with $\mathrm{X}$-gal solution $(0.05 \%(\mathrm{v} / \mathrm{v})$ 5-bromo-4-chloro-3-indolyl- $\beta$-D-galactoside (X-gal; Nacalai Tesque, Kyoto, Japan), $1 \mathrm{mM} \mathrm{MgCl}_{2}, 150 \mathrm{mM}$ $\mathrm{NaCl}, 3 \mathrm{mM} \mathrm{K} \mathrm{K}_{4}\left[\mathrm{Fe}(\mathrm{CN})_{6}\right], 3 \mathrm{mM} \mathrm{K} \mathrm{K}_{3}\left[\mathrm{Fe}(\mathrm{CN})_{6}\right], 60 \mathrm{mM} \mathrm{Na}{ }_{2} \mathrm{HPO}_{4}$, $40 \mathrm{mM} \mathrm{NaH} \mathrm{PO}_{4}$, and $0.1 \%$ Triton X-100). After $12 \mathrm{~h}$ of incubation at $37^{\circ} \mathrm{C}$, the reaction was terminated by replacing the solution with PBS [33].

Fluorescence microscopic observation and immunohistochemistry. After perfusion with saline, the heart was fixed with $4 \%$ paraformaldehyde and dehydrated in sucrose solution. The specimens were embedded in OCT compound and immediately frozen at $-80^{\circ} \mathrm{C}$. Serial sections $10 \mu \mathrm{m}$ thick were cut on a cryostat and observed under a fluorescence microscope with excitation at $488 \mathrm{~nm}$. The cryosections were stained with anti-GFP antibody (Molecular Probes, Leiden, The Netherlands) and visualized using the avidin/biotin/peroxidase method (Vector Laboratories, Burlingame, CA).

Statistical analysis. Differences among continuous variables were tested by Student's $t$ test for paired and unpaired observations and by ANOVA with Fisher's PLSD correction for repeated comparisons. A value of $P<0.05$ was considered to be statistically significant. 


\section{Results}

\section{Influence of sonoporation to the heart}

A mixture of plasmid DNA and BR14 microbubble was injected into the LV of mice. Echocardiographic examinations were performed before and after the injection. The myocardium and the LV lumen were separated by endocardium that was visible before the treatment (Fig. 1Aa). After BR14 injection, the myocardium and the LV cavity showed comparable echo intensities, and the boundary between them was unclear (Fig. 1Ab). The hearts of the mice were sonicated under several conditions. After continuous sonication at $2.0 \mathrm{~W} / \mathrm{cm}^{2}$ and $50 \%$ PDR $(2.0 \mathrm{~W} / 50 \%$ PDR) for $60 \mathrm{~s}$, most of the BR14 was disrupted, while a few residual circulating microbubbles were seen in the ventricle (Figs. 1Ac and Ad, arrowheads). The echocardiographic appearance subsequently became normal thereafter (data not shown).

Electrocardiographic examination was also performed to assess the influence of sonotransfection on the murine heart. Arrhythmia was seen in the mice exposed to ultrasound at $2.0 \mathrm{~W} / 50 \%$ PDR after pDNA and BR14 had been injected into the LV, but they all quickly recovered from the same. Fig. 1B shows representative ECG changes before, during, and after the manipulation. Compared with the ECG profile before sonication (Fig. 1Ba), the QRS complex was prolonged immediately after the initiation of the ultrasound exposure (Fig. 1Bb). Thirty seconds later, the mice showed atrioventricular (AV) block (Fig. 1Bc), which persisted thereafter (Fig. 1Bd). Two minutes after termination of the sonication, the heart rate suddenly recovered to baseline (Figs. 1Be and Bf). In other cases, the AV block continued for shorter periods, ranging from $10 \mathrm{~s}$ to $2 \mathrm{~min}$.

Echocardiographic analyses were performed to evaluate the LV contractility before and after sonoporation-mediated transfection (LV method, $2.0 \mathrm{~W} / 50 \%$ PDR). As shown in Table 1, no significant change was observed for the parameters, Dd, Ds, IVS, PW, FS, and EF. The results indicated that the contractility of LV was not affected, even though its apex was punc-

Table 1

Echocardiographic analyses at pre- and post-sonoporation

\begin{tabular}{llll}
\hline & Pre-transfection $(n=5)$ & Post-transfection $(n=5)$ & $P$ \\
\hline BW $(\mathrm{g})$ & $22.8 \pm 0.37$ & $22.8 \pm 0.20$ & $\mathrm{~ns}$ \\
IVSTd $(\mathrm{mm})$ & $0.54 \pm 0.02$ & $0.60 \pm 0.03$ & $\mathrm{~ns}$ \\
PWTd (mm) & $0.62 \pm 0.02$ & $0.58 \pm 0.02$ & $\mathrm{~ns}$ \\
LVIDd (mm) & $3.24 \pm 0.02$ & $3.28 \pm 0.05$ & $\mathrm{~ns}$ \\
LVIDs (mm) & $2.34 \pm 0.04$ & $2.40 \pm 0.06$ & $\mathrm{~ns}$ \\
EF (\%) & $60.8 \pm 2.15$ & $61.0 \pm 1.90$ & $\mathrm{~ns}$ \\
FS $(\%)$ & $26.8 \pm 1.39$ & $27.0 \pm 1.27$ & $\mathrm{~ns}$ \\
\hline
\end{tabular}

Cardiac function was not significantly affected by sonoporation-mediated transfection of pDNA/BR14. M-mode echocardiographic analysis was performed on mice before and 4 days after sonoporation at $2.0 \mathrm{~W} / 50 \%$ PDR. BW, body weight; IVSTd, interventricular septum thickness; PWTd, LV posterior wall thickness; LVIDd, LV internal dimensions at end diastole; LVIDs, LV internal dimensions at end systole; EF, ejection fraction; FS, fractional shortening. Data are expressed as means $\pm \mathrm{SE}$.
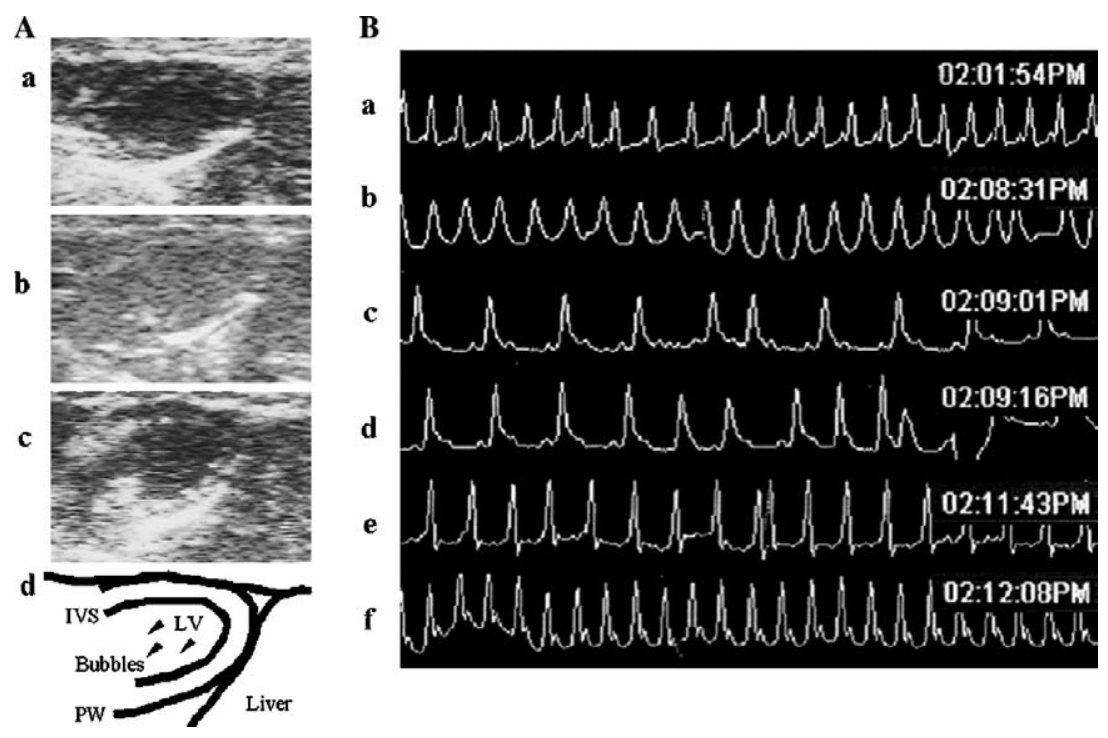

Fig. 1. (A) BR14 microbubbles were mostly disrupted by sonoporation, while some remained intact. C57BL/6 mice were injected with pGEG.GL3/ BR14 into the LV. Shown are representative echocardiographic images before (a), immediately after (b), and $60 \mathrm{~s}$ after (c) the sonication at $2.0 \mathrm{~W} /$ $50 \%$ PDR, while an echocardiographic image of (c) is illustrated schematically in (d). IVS, interventricular septum; PW, posterior wall. Arrowheads, intact microbubbles. (B) The transient arrhythmia caused by sonoporation. Mice received LV injection with pGEG.GL3/BR14, and sonoporation at $2.0 \mathrm{~W} / 50 \%$ PDR was performed. Shown are representative ECG records before (a), and $0 \mathrm{~s}(\mathrm{~b}), 30 \mathrm{~s} \mathrm{(c),} 45 \mathrm{~s}$ (d), $3 \mathrm{~min}(\mathrm{e})$, and $3.5 \mathrm{~min}(\mathrm{f})$ after the initiation of sonoporation. 
tured on injection. The body weight of the mice did not significantly change following the sonoporation-mediated transfection.

\section{Sonoporation remarkably facilitated cardiac gene transfer in mice}

A mixture of pGEG.GL3 and BR14 was injected into the mice via their TV or LV. Ultrasound was directed at the chest at various output intensities and PDRs, and 2-7 days later Luc activity in the heart was measured. Representative data are shown in Fig. 2. When the mixture was injected into the $\mathrm{LV}$, sonication at $2.0 \mathrm{~W} / 50 \%$ PDR induced a significantly higher Luc activity on day 4 compared with that at $1.0 \mathrm{~W} / 20 \%$ PDR (approximately 46 -fold) or $1.0 \mathrm{~W} / 50 \%$ PDR (approximately 13 fold). Transfection into the heart was more efficient when the mixture was injected into the LV than when it was injected into the TV. For example, compared with injection into the TV, expression was 67 -fold higher 4 days following injection into the $\mathrm{LV}$ with sonoporation at $2.0 \mathrm{~W} / 50 \%$ PDR. Optimal transfection was achieved by intra-LV injection and ultrasonication at $2.0 \mathrm{~W} /$ $50 \%$ PDR, which resulted in Luc activity as high as approximately $6.0 \times 10^{4} \mathrm{RLU} / \mathrm{mg}$ protein/10 s on day 4 , although the transgene activity was significantly decreased by day 7 .

Transgene product was predominantly detected at subendocardial layer of the myocardium and anterior-septal wall of $L V$

To identify the localization of expression of the transferred gene, the heart was transfected with pGEG. $\beta$ or pGEG.EGFP under the optimal conditions determined as described above. X-gal staining of the
pGEG. $\beta$-transfected heart demonstrated that the $\beta$-gal was significantly expressed in the subendocardial layer of the LV and a part of the right ventricle. The strongest expression was detected at the anterior-septal wall of the LV that had faced the ultrasound probe (Fig. 3B). The pGEG.EGFP-transfected heart was examined histologically by fluorescence microscopy. The green fluorescence was observed in cardiomyocytes in the subendocardial layer (Figs. 3D and F). Consistent results were obtained by immunohistochemical staining of the pGEG.EGFPtransfected heart (data not shown). These findings indicated that transgene product was expressed in the subendocardial layer as well as in the anterior-septal wall of the LV.

Heart-specific transfection was achieved by sonoporationbased gene delivery

To investigate whether the present protocol allowed gene transfection in organs other than the heart, pGEG.GL3 was transfected as described above and the levels of Luc activity were determined quantitatively in lung, liver, kidney, spleen, intestine, ovary, and brain. Luc activity was not significantly increased in any of the organs tested (Fig. 4), indicating that the transfection procedure could deliver genes only to the heart.

\section{Sonotransduction of siRNA induced specific gene} silencing in coronary artery

We investigated whether the sonoporation-assisted DNA delivery procedure is also applicable to delivering siRNA into the heart. siRNA duplex specific for EGFP is capable of silencing the fluorescent protein in vivo, as demonstrated by preliminary experiments in which the siRNA was injected intravenously into EGFP TGM

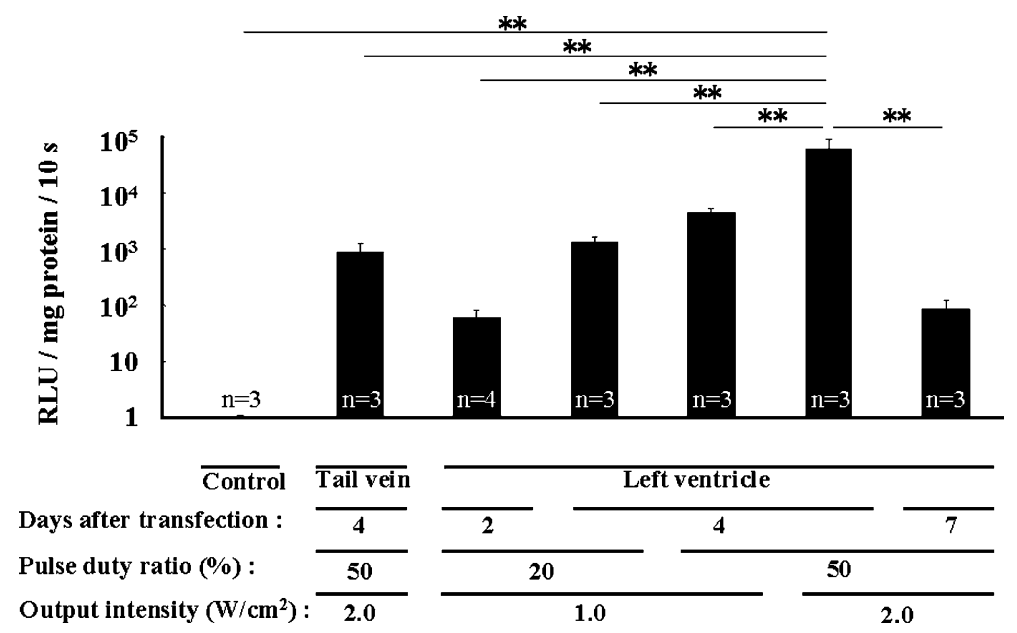

Fig. 2. Ultrasound-mediated transfection of pDNA under various conditions. pGEG.GL3/BR14 solution was injected into mice via the LV or TV route, and the heart was sonicated under the indicated conditions. Two, four, or seven days later, mice were sacrificed and Luc activities in the heart extracts were measured. Bars, SE. ${ }^{* *} P<0.01$. 

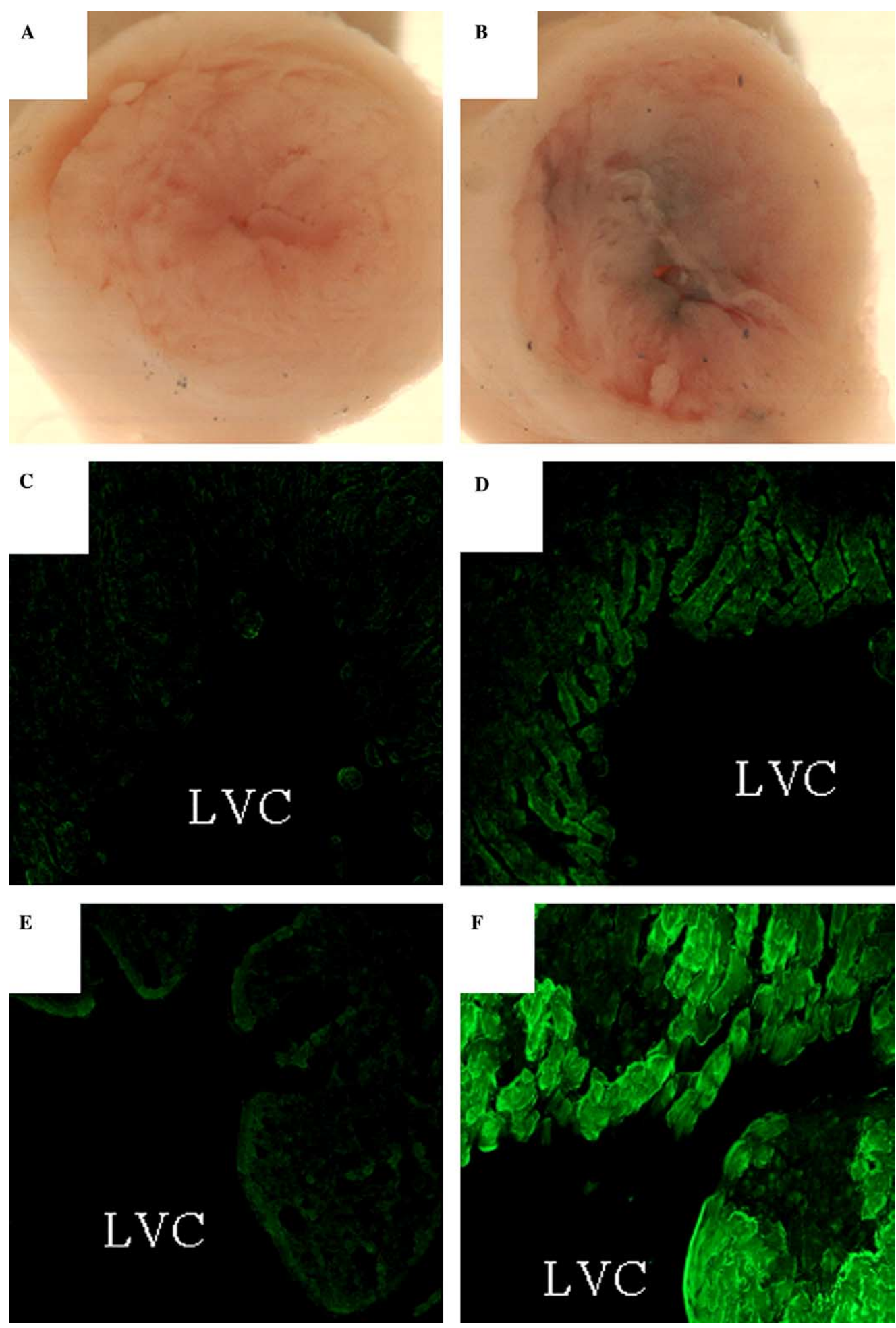

Fig. 3. Expression of transgene product in cardiomyocytes at the subendocardial layer and anterior-septal wall. Mice were given an intra-LV injection with pGEG. $\beta /$ BR 14, and sonication was performed at $2.0 \mathrm{~W} / 50 \% \mathrm{PDR}$ (B, D, and F). Control mice were left untreated (A, C, and E). Four days after the transfection, the hearts were excised. Shown are stereomicroscopic images of X-gal-stained cross-sections (A,B) and fluorescence microscopic images of cryosections (C-F). LVC, left ventricular cavity. Original magnifications were 4× (A,B), 40× (C,D), and 100× (E,F).

and expression of the transgene product was substantially suppressed in the hepatic parenchymal cells (Fig. 5B). siRNA was transfected into EGFP TGM, and the heart sections were observed by a fluorescence microscope.
Representative findings are shown in Figs. 5C-F. In the control mice that received an injection of BR14 but not siRNA, the sonoporation procedure failed to affect EGFP expression in cardiomyocytes or coronary 


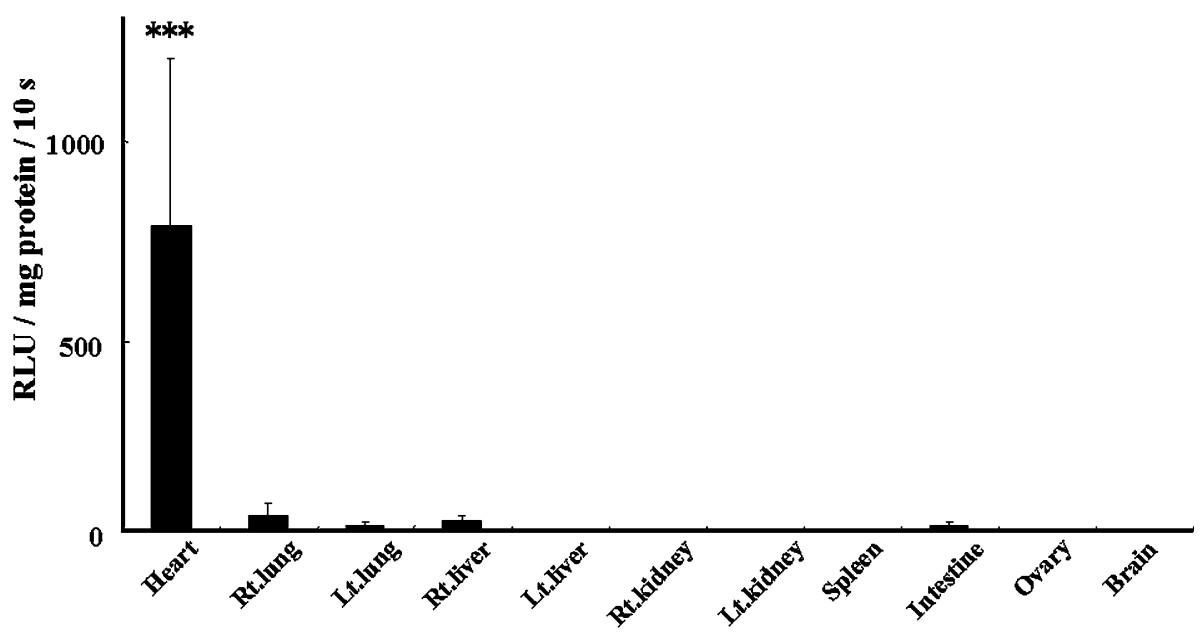

Fig. 4. Heart-specific pDNA transfer obtained by sonoporation-mediated transfection. Mice received an injection of pGEG.GL3/BR14 into the LV, and sonication was performed at $1.0 \mathrm{~W} / 20 \%$ PDR. Four days later, the indicated organs were excised and Luc activity in each tissue extract was measured. Bars, SE. ${ }^{* * *} P<0.001$ vs other organs $(n=3)$.

vessels (Figs. 5C and E). Intra-LV co-injection of siRNA-GFP and BR14 in combination with sonication at $1.0 \mathrm{~W} / 10 \%$ PDR resulted in a substantial reduction of EGFP expression in the coronary artery wall $48 \mathrm{~h}$ after transduction, while cardiomyocytes were not influenced in terms of the intensity of the green fluorescence (Figs. 5D and F). The silencing of EGFP expression was detected not only at the main trunk of the left and right coronary arteries, but also at peripheral branches of the coronary arterial wall that could be identified by the structure of their vascular lumen. Similar results were also obtained by sonoporation performed under two other conditions, i.e., $1.0 \mathrm{~W} / 10 \%$ PDR and $2.0 \mathrm{~W} / 50 \%$ PDR (data not shown).

\section{Discussion}

Intra-LV injection of a mixture of an expression vector and the micobubble agent BR14 and concomitant ultrasonication resulted in significant expression of the marker gene in cardiomyocytes in the subendocardial layer and the antero-septal wall of the murine heart. BR14 also facilitated sonoporation-mediated transfer of siRNA duplex into the coronary artery, resulting in substantial gene silencing in vivo. Our study suggests an improved methodology for achieving safe and efficient genetic modification in the heart, in both gain-offunction and loss-of-function manners.

The present study also indicates that sonoporationmediated pDNA transfer is strongly enhanced by the third-generation microbubble, BR14. BR14 is a negatively charged ultrasound contrast agent consisting of perfluorobutane-containing microbubbles stabilized by a phospholipid monolayer [20,34,35]. We also compared BR14 with Optison in sonoporation-mediated transfection into murine heart. An intra-TV injection of
$500 \mu \mathrm{g}$ pGEG.GL3/Optison and sonoporation at $2.0 \mathrm{~W} / 50 \%$ PDR resulted in Luc expression in the cardiac muscle at a level similar to that when BR14 was used $(1330 \pm 310 \mathrm{RLU} / \mathrm{mg}$ protein/10 s on day $4(n=9))$. However, some mice died immediately after receiving an intra-TV injection of Optison even without the sonoporation. Although Optison has been shown to be a useful agent for ultrasound-based transfection in rats and larger animals $[9,12-14,17,18]$, BR 14 may be more suitable in mice because a TV injection of BR14 did not kill any mice in the present study. The difference in results may be due to difference in the size of the agents: the mean diameter of BR14 is $2.5-3.0 \mu \mathrm{m}$, which is small compared with its predecessors $[17,35,36]$. The diameter of erythrocytes in C57BL/6 mice is about a half of that in humans (MCV $44.8 \pm 0.26 \mathrm{fl}, \mathrm{MCH} 15.6 \pm 0.49 \mathrm{pg}$, $\mathrm{MCHC} 34.7 \pm 1.12 \mathrm{~g} / \mathrm{dl}$ ). Fisher et al. [36] examined the distribution of microbubbles intravenously administered into C57BL-6 mice. Intravital microscopic analyses demonstrated that neutral microbubbles larger than $4 \mu \mathrm{m}$ in diameter persisted in capillaries in a sizedependent manner. Although the mean diameter of Optison is $2.0-4.5 \mu \mathrm{m}$, it contains bubbles up to $32 \mu \mathrm{m}$ in diameter. These large bubbles may cause lethal embolisms in some vital organs in mice.

With regard to the routes of injection of pDNA/ BR14, a higher rate of pDNA transfection was obtained by injection into the LV than by injection into the TV. These results suggest that high concentrations of pDNA and microbubble in coronary circulation are required at the time of sonication to achieve efficient sonoporationbased transfection.

In our study, pDNA could have reached the myocardium via 2 routes, i.e., directly from the LV cavity or through the coronary capillaries. This may explain why pDNA was predominantly delivered into the myocardium in the subendocardial layer and the anterior-septal 

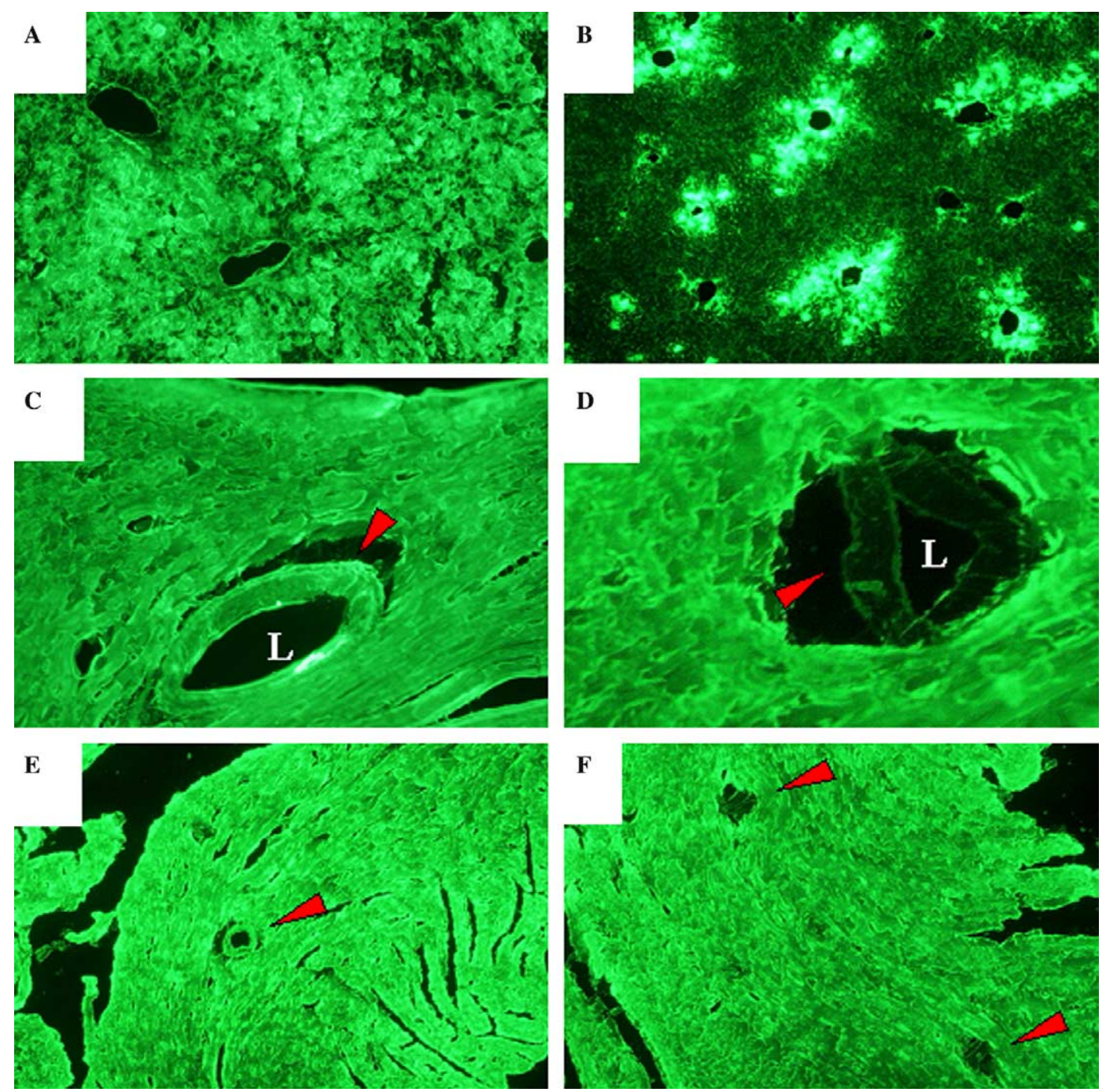

Fig. 5. Sonoporation-based transduction of siRNA silenced EGFP expression in the coronary arterial wall. (A) Fluorescence microscopic image of a liver section of EGFP-TGM. (B) EGFP-TGM were given a rapid tail vein injection with siRNA-GFP. Two days later, cryosections of the liver were observed under fluorescence microscope. (C-F) EGFP-TGM received an intra-LV injection of BR14 (C,E) or siRNA-GFP/BR14 (D,F), and sonication was performed at $1.0 \mathrm{~W} / 10 \%$ PDR. Two days later, cryosections of the heart were observed under fluorescence microscope. Arrowheads in $(C, D)$ indicate the left coronary artery, while those in $(\mathrm{E}, \mathrm{F})$ indicate the septal branches. L, lumen of arteries. Original magnifications were $100 \times$ $(\mathrm{A}, \mathrm{B}), 200 \times(\mathrm{C}, \mathrm{D})$, and $40 \times(\mathrm{E}, \mathrm{F})$.

wall. Chen et al. [16] showed that the rate of pDNA transfection was significantly diminished by destroying microbubbles in the LV cavity before their entrance into the coronary microcirculation. So, presumably, the quantity of the pDNA transported via the coronary capillaries was greater than that delivered directly through the LV.

The present results showed that cardiomyocytes in the anterior-septal wall expressed the transgene more strongly than those in the inferior-posterior wall. The ultrasound beam is probably attenuated during passage through the cardiac muscle layers, so that the transfection efficiency may be inversely proportional to the distance from the probe. These results are consistent with the report by Chen et al. [16], who demonstrated that gene expression in the anterior wall was 2- to 2.5 -fold greater than that in the posterior wall in rat heart transfected with pDNA by their sonoporation-mediated transduction protocols.

In the present study, maximum expression of transgene was obtained 4 days after transfection. This is consistent with a previous study in which expression levels peaked on day 4 following sonoporation-mediated transfer of pDNA and Optison into rat hearts [15].

In some previous studies, ultrasound-mediated destruction of Optison and other microbubbles induced rupture of microvessels at the sonicated site of the target organs $[37,38]$. In our experiments, a careful microscopic study did not demonstrate the RBC extravasation into the myocardium (data not shown). This is consistent 
with echocardiographic examinations which demonstrated that cardiac contractility was not significantly affected after sonoporation (Table 1).

Some previous studies indicated that RNAi was induced in vitro into rat cardiomyocytes [39] as well as in human coronary vascular smooth muscle cells [40] in culture. We also demonstrated successful sequencespecific knockdown of endogenous gene expression in vivo in the coronary artery of adult mice using siRNA. RNAi may offer novel strategies for therapeutic molecular targeting, enabling artificial regulation of proteins that are responsible for the pathogenesis and/or progression of coronary diseases. For example, specific silencing of $\mathrm{NF}-\kappa \mathrm{B}$ or platelet-derived growth factor (PDGF) A-chain may suppress neointimal formation in coronary vasculature, which may have prophylactic implications for restenosis after coronary intervention as well as for the progression of atherosclerosis. These concepts are supported by previous studies in which balloon-injured artery models of animals were treated with decoy oligonucleotide that suppresses NF- $\mathrm{BB}$ activity [41] or a PDGF-targeting ribozyme [42].

It is noteworthy that pDNA was transfected effectively into cardiomyocytes, while the siRNA was transduced into coronary vascular wall. Although the precise mechanisms underlying these phenomena remain unclear, we propose the following explanation. Ultrasound-mediated cavitation may damage endothelial cells in both arteries and capillaries. Due to its small size, siRNA may easily diffuse from the bloodstream into tissue via endothelium when passing through the coronary arteries and arterioles, resulting in efficient transduction into arterial/arteriolar walls including the smooth muscle cells. Because pDNA permeates endothelium more slowly, transduction occurs less readily during its passage through arterioles. Instead, the pDNA is transduced predominantly at the capillary bed where cavitation from transient microbubbles is more intense. As a result, pDNA may be transfected into cardiomyocytes residing immediately beyond the extremely thin capillary wall.

To obtain further higher transduction efficacy, the following may be employed in combination with the sonoporatoin. Administration with the vascular endothelial growth factor (VEGF) may facilitate transfer of genetic materials through the endothelium, due to an increase in permeability of the endothelial cell membrane [43]. ECG-triggered sonication may also be effective because ultrasound-based cavitation can be generated precisely at the diastolic phase, when the coronary blood flow is higher than in the systolic phase. Use of RNase inhibitor [25] may prevent degradation of siRNA.

Kunichika et al. [20] showed that BR14 highlights ischemic risk areas in myocardium as a hyperenhanced "hot spot" when myocardial contrast echocardiography is performed following coronary reperfusion. Therefore, sonoporation using BR14 may enable targeted gene delivery to ischemic cardiomyocytes in patients with myocardial infarction, specifically delivering therapeutic nucleic acids into damaged cells.

Finally, it should be emphasized that the present procedure enables gene transfer and knockdown in the murine heart, although previous studies have documented ultrasound-assisted transfection into the hearts of larger animals, including rats $[10,16]$ and dogs [11]. The mouse is a useful animal for post-genomic experimental cardiology. Particularly, a variety of transgenic and gene out mice have been established, providing extremely useful tools to elucidate the molecular mechanisms of cardiovascular disorders. The present method may be applied to these genetically engineered mice, providing a suitable technology to promote functional genomic analyses, as well as to examine candidate molecules for therapeutic molecular targeting for cardiovascular diseases.

\section{Acknowledgment}

This work was supported by a Grant-in-Aid for scientific research from the Japanese Ministry of Education, Culture, Sports, Science and Technology.

\section{References}

[1] M. Iwatate, Y. Gu, T. Dieterle, Y. Iwanaga, K.L. Peterson, M. Hoshijima, K.R. Chien, J. Ross, In vivo high-efficiency transcoronary gene delivery and Cre-LoxP gene switching in the adult mouse heart, Gene Ther. 10 (2003) 1814-1820.

[2] H.C. Champion, D. Georgakopoulos, S. Haldar, L. Wang, Y. Wang, D.A. Kass, Robust adenoviral and adeno-associated viral gene transfer to the in vivo murine heart: application to study of phospholamban physiology, Circulation 108 (2003) 2790-2797.

[3] K. Li, R.E. Welikson, K.L. Vikstrom, L.A. Leinwand, Direct gene transfer into the mouse heart, J. Mol. Cell. Cardiol. 29 (1997) 1499-1504.

[4] H. Lin, M.S. Parmacek, G. Morle, S. Bolling, J.M. Leiden, Expression of recombinant genes in myocardium in vivo after direct injection of DNA, Circulation 82 (1990) 2217-2221.

[5] R.N. Kitsis, P.M. Buttrick, E.M. McNally, M.L. Kaplan, L.A. Leinwand, Hormonal modulation of a gene injected into rat heart in vivo, Proc. Natl. Acad. Sci. USA 88 (1991) 4138-4142.

[6] K. Tomiyasu, Y. Oda, M. Nomura, E. Satoh, S. Fushiki, J. Imanishi, M. Kondo, O. Mazda, Direct intra-cardiomuscular transfer of beta2-adrenergic receptor gene augments cardiac output in cardiomyopathic hamsters, Gene Ther. 7 (2000) 20872093.

[7] K. Ogawa, K. Tachibana, T. Uchida, T. Tai, N. Yamashita, N. Tsujita, R. Miyauchi, High-resolution scanning electron microscopic evaluation of cell-membrane porosity by ultrasound, Med. Electron Microsc. 34 (2001) 249-253.

[8] D.L. Miller, S.V. Pislaru, J.E. Greenleaf, Sonoporation: mechanical DNA delivery by ultrasonic cavitation, Somat. Cell Mol. Genet. 27 (2002) 115-134. 
[9] T. Li, K. Tachibana, M. Kuroki, M. Kuroki, Gene transfer with echo-enhanced contrast agents: comparison between Albunex, Optison, and Levovist in mice-initial results, Radiology 229 (2003) 423-428.

[10] R.V. Shohet, S. Chen, Y.T. Zhou, Z. Wang, R.S. Meidell, R.H. Unger, P.A. Grayburn, Echocardiographic destruction of albumin microbubbles directs gene delivery to the myocardium, Circulation 101 (2000) 2554-2556.

[11] M. Vannan, T. McCreery, P. Li, Z. Han, E. Unger, B. Kuersten, E. Nabel, S. Rajagopalan, Ultrasound-mediated transfection of canine myocardium by intravenous administration of cationic microbubble-linked plasmid DNA, J. Am. Soc. Echocardiogr. 15 (2002) 214-218.

[12] M. Blomley, Which US microbubble contrast agent is best for gene therapy? Radiology 229 (2003) 297-298.

[13] S. Miura, K. Tachibana, T. Okamoto, K. Saku, In vitro transfer of antisense oligodeoxynucleotides into coronary endothelial cells by ultrasound, Biochem. Biophys. Res. Commun. 298 (2002) 587590.

[14] Y. Taniyama, K. Tachibana, K. Hiraoka, T. Namba, K. Yamasaki, N. Hashiya, M. Aoki, T. Ogihara, K. Yasufumi, R. Morishita, Local delivery of plasmid DNA into rat carotid artery using ultrasound, Circulation 105 (2002) 1233-1239.

[15] R. Bekeredjian, S. Chen, P.A. Frenkel, P.A. Grayburn, R.V. Shohet, Ultrasound-targeted microbubble destruction can repeatedly direct highly specific plasmid expression to the heart, Circulation 108 (2003) 1022-1026.

[16] S. Chen, R.V. Shohet, R. Bekeredjian, P. Frenkel, P.A. Grayburn, Optimization of ultrasound parameters for cardiac gene delivery of adenoviral or plasmid deoxyribonucleic acid by ultrasoundtargeted microbubble destruction, J. Am. Coll. Cardiol. 42 (2003) 301-308.

[17] S. Mayer, P.A. Grayburn, Myocardial contrast agents: recent advances and future directions, Prog. Cardiovasc. Dis. 44 (2001) 33-44.

[18] R. Bekeredjian, P.A. Grayburn, R.V. Shohet, Use of ultrasound contrast agents for gene or drug delivery in cardiovascular medicine, J. Am. Coll. Cardiol. 45 (2005) 329-335.

[19] N.G. Fisher, H. Leong-Poi, T. Sakuma, S.J. Rim, J.P. Bin, S. Kaul, Detection of coronary stenosis and myocardial viability using a single intravenous bolus injection of BR14, J. Am. Coll. Cardiol. 39 (2002) 523-529.

[20] H. Kunichika, B. Peters, B. Cotter, H. Masugata, N. Kunichika, P.L. Wolf, A.N. DeMaria, Visualization of risk-area myocardium as a high-intensity, hyperenhanced "hot spot" by myocardial contrast echocardiography following coronary reperfusion: quantitative analysis, J. Am. Coll. Cardiol. 42 (2003) $552-557$.

[21] Y. Sakakima, S. Hayashi, Y. Yagi, A. Hayakawa, K. Tachibana, A. Nakao, Gene therapy for hepatocellular carcinoma using sonoporation enhanced by contrast agents, Cancer Gene Ther. (in press).

[22] E.F. Akowuah, C. Gray, A. Lawrie, P.J. Sheridan, C.H. Su, T. Bettinger, A.F. Brisken, J. Gunn, D.C. Crossman, S.E. Francis, A.H. Baker, C.M. Newman, Ultrasound-mediated delivery of TIMP-3 plasmid DNA into saphenous vein leads to increased lumen size in a porcine interposition graft model, Gene Ther. 12 (2005) 1154-1157.

[23] G.J. Hannon, RNA interference, Nature 418 (2002) 244-251.

[24] J. Downward, RNA interference, Br. Med. J. 328 (2004) 12451248.

[25] A.P. McCaffrey, L. Meuse, T.T. Pham, D.S. Conklin, G.J. Hannon, M.A. Kay, RNA interference in adult mice, Nature 418 (2002) 38-39.

[26] D.L. Lewis, J.E. Hagstrom, A.G. Loomis, J.A. Wolff, H. Herweijer, Efficient delivery of siRNA for inhibition of gene expression in postnatal mice, Nat. Genet. 32 (2002) 107-108.
[27] T. Kishida, H. Asada, S. Gojo, S. Ohashi, M. Shin-Ya, K. Yasutomi, R. Terauchi, K.A. Takahashi, T. Kubo, J. Imanishi, O. Mazda, Sequence-specific gene silencing in murine muscle induced by electroporation-mediated transfer of short interfering RNA, J. Gene. Med. 6 (2004) 105-110.

[28] F.D. Cui, T. Kishida, S. Ohashi, H. Asada, K. Yasutomi, E. Satoh, T. Kubo, S. Fushiki, J. Imanishi, O. Mazda, Highly efficient gene transfer into murine liver achieved by intravenous administration of naked Epstein-Barr virus (EBV)-based plasmid vectors, Gene Ther. 8 (2001) 1508-1513.

[29] T. Kishida, H. Asada, Y. Itokawa, F.D. Cui, M. Shin-Ya, S. Gojo, K. Yasutomi, Y. Ueda, H. Yamagishi, J. Imanishi, O. Mazda, Interleukin (IL)-21 and IL-15 genetic transfer synergistically augments therapeutic antitumor immunity and promotes regression of metastatic lymphoma, Mol. Ther. 8 (2003) 552-558.

[30] H. Nakanishi, O. Mazda, E. Satoh, H. Asada, H. Morioka, T. Kishida, M. Nakao, Y. Mizutani, A. Kawauchi, M. Kita, J. Imanishi, T. Miki, Nonviral genetic transfer of Fas ligand induced significant growth suppression and apoptotic tumor cell death in prostate cancer in vivo, Gene Ther. 10 (2003) 434 442.

[31] O. Mazda, Improvement of nonviral gene therapy by EpsteinBarr virus (EBV)-based plasmid vectors, Curr. Gene. Ther. 2 (2002) 379-392.

[32] S. Ohashi, T. Kubo, T. Kishida, T. Ikeda, K. Takahashi, Y, Arai, R. Terauchi, H. Asada, J. Imanishi, O. Mazda, Successful genetic transduction in vivo into synovium by means of electroporation, Biochem. Biophys. Res. Commun. 293 (2002) $1530-1535$.

[33] K. Tomiyasu, E. Satoh, Y. Oda, K. Nishizaki, M. Kondo, J. Imanishi, O. Mazda, Gene transfer in vitro and in vivo with Epstein-Barr virus-based episomal vector results in markedly high transient expression in rodent cells, Biochem. Biophys. Res. Commun. 253 (1998) 733-738.

[34] H. Takeuchi, K. Ohmori, I. Kondo, K. Shinomiya, A. Oshita, Y. Takagi, J. Yoshida, K. Mizushige, M. Kohno, Interaction with leukocytes: phospholipid-stabilized versus albumin-shell microbubbles, Radiology 230 (2004) 735-742.

[35] N.G. Fisher, J.P. Christiansen, H. Leong-Poi, A.R. Jayaweera, J.R. Lindner, S. Kaul, Myocardial and microcirculatory kinetics of BR14, a novel third-generation intravenous ultrasound contrast agent, J. Am. Coll. Cardiol. 39 (2002) 530-537.

[36] N.G. Fisher, J.P. Christiansen, A. Klibanov, R.P. Taylor, S. Kaul, J.R. Lindner, Influence of microbubble surface charge on capillary transit and myocardial contrast enhancement, J. Am. Coll. Cardiol. 40 (2002) 811-819.

[37] D.L. Miller, J. Quddus, Diagnostic ultrasound activation of contrast agent gas bodies induces capillary rupture in mice, Proc. Natl. Acad. Sci. USA 97 (2000) 10179-10184.

[38] R.J. Price, D.M. Skyba, S. Kaul, T.C. Skalak, Delivery of colloidal particles and red blood cells to tissue through microvessel ruptures created by targeted microbubble destruction with ultrasound, Circulation 98 (1998) 1264-1267.

[39] A. Watanabe, M. Arai, M. Yamazaki, N. Koitabashi, F. Wuytack, M. Kurabayashi, Phospholamban ablation by RNA interference increases $\mathrm{Ca}^{2+}$ uptake into rat cardiac myocyte sarcoplasmic reticulum, J. Mol. Cell. Cardiol. 37 (2004) 691698.

[40] F. Blaschke, D. Bruemmer, F. Yin, Y. Takata, W. Wang, M.C. Fishbein, T. Okura, J. Higaki, K. Graf, E. Fleck, W.A. Hsueh, R.E. Law, C-reactive protein induces apoptosis in human coronary vascular smooth muscle cells, Circulation 110 (2004) 579-587.

[41] K. Yamasaki, T. Asai, M. Shimizu, M. Aoki, N. Hashiya, H. Sakonjo, H. Makino, Y. Kaneda, T. Ogihara, R. Morishita, Inhibition of NFkappaB activation using cis-element 'decoy' of NFkappaB binding site reduces neointimal formation in porcine balloon-injured coronary artery model, Gene Ther. 10 (2003) 356-364. 
[42] M. Kotani, N. Fukuda, H. Ando, W.Y. Hu, S. Kunimoto, S. Saito, K. Kanmatsuse, Chimeric DNA-RNA hammerhead ribozyme targeting PDGF A-chain mRNA specifically inhibits neointima formation in rat carotid artery after balloon injury, Cardiovasc. Res. 57 (2003) 265-276.
[43] P. Gregorevic, M.J. Blankinship, J.M. Allen, R.W. Crawford, L. Meuse, D.G. Miller, D.W. Russell, J.S. Chamberlain, Systemic delivery of genes to striated muscles using adeno-associated viral vectors, Nat. Med. 10 (2004) 828-834. 